\title{
LOWER BOUNDS FOR THE PRINCIPAL GENUS OF DEFINITE BINARY QUADRATIC FORMS
}

\author{
KIMBERLY HOPKINS AND JEFFREY STOPPLE
}

\begin{abstract}
We apply Tatuzawa's version of Siegel's theorem to derive two lower bounds on the size of the principal genus of positive definite binary quadratic forms.
\end{abstract}

Introduction. Suppose $-D<0$ is a fundamental discriminant. By genus theory we have an exact sequence for the class group $\mathcal{C}(-D)$ of positive definite binary quadratic forms:

$$
\mathcal{P}(-D) \stackrel{\text { def. }}{=} \mathcal{C}(-D)^{2} \hookrightarrow \mathcal{C}(-D) \rightarrow \mathcal{C}(-D) / \mathcal{C}(-D)^{2} \simeq(\mathbb{Z} / 2)^{g-1},
$$

where $D$ is divisible by $g$ primary discriminants (i.e., $D$ has $g$ distinct prime factors). Let $p(-D)$ denote the cardinality of the principal genus $\mathcal{P}(-D)$. The genera of forms are the cosets modulo the principal genus, and thus $p(-D)$ is the number of classes of forms in each genus. The study of this invariant of the class group is as old as the study of the class number $h(-D)$ itself. Indeed, Gauss wrote in [3, Art. 303]

... Further, the series of [discriminants] corresponding to the same given classification (i.e. the given number of both genera and classes) always seems to terminate with a finite number . . However, rigorous proofs of these observations seem to be very difficult.

Theorems about $h(-D)$ have usually been closely followed with an analogous result for $p(-D)$. When Heilbronn showed [4] that $h(-D) \rightarrow$ $\infty$ as $D \rightarrow \infty$, Chowla [1] showed that $p(-D) \rightarrow \infty$ as $D \rightarrow \infty$. Chowla's result appeared in the same journal issue as Heilbronn's, and his enthusiasm to appear in print detracted from the exposition - the crucial estimate appears in a footnote on the last page without proof. An elegant proof of Chowla's theorem is given by Narkiewicz in [8, Prop 8.8 p. 458].

Similarly, the Heilbronn-Linfoot result [5] that $h(-D)>1$ if $D>$ 163 , with at most one possible exception was matched by Weinberger's result [14] that $p(-D)>1$ if $D>5460$ with at most one possible

1991 Mathematics Subject Classification. 11M20,11R29. 
exception. On the other hand, Oesterlé's [9] exposition of the GoldfeldGross-Zagier bound for $h(-D)$ already contains the observation that the result was not strong enough to give any information about $p(-D)$.

In [13] Tatuzawa proved a version of Siegel's theorem: for every $\epsilon$ there is an explicit constant $C(\epsilon)$ so that

$$
h(-D)>C(\epsilon) D^{1 / 2-\epsilon}
$$

with at most one exceptional discriminant $-D$. This result has never been adapted to the study of the principal genus. It is easily done; the proofs are not difficult so it is worthwhile filling this gap in the literature. We present two versions. The first version gives, for each $n \geq 4$, a bound which involves only elementary functions. The second version contains a transcendental function (the Lambert $W$ function discussed below); for each fixed $n$ the first version is stronger on an interval of $D$, but the second is stronger as $D \rightarrow \infty$. (N.B. The constants in Tatuzawa's result have been improved in [6] and [7]; these could be applied at the expense of slightly more complicated statements.)

Notation. We will always assume that $g \geq 2$, for if $g=1$ then $-D=$ $-4,-8$, or $-q$ with $q \equiv 3 \bmod 4$ a prime. In this last case $p(-q)=$ $h(-q)$ and Tatuzawa's theorem [13] applies directly.

\section{FIRST VERSION}

Theorem 1. Let $n \geq 4$ be any natural number. If $0<\epsilon<1 / 2$ and $D>\max (\exp (1 / \epsilon), \exp (11.2))$, then with at most one exception

$$
p(-D)>\frac{1.31 \epsilon}{\pi} \cdot \frac{D^{1 / 2-\epsilon-1 / n}}{f(n)},
$$

where

$$
f(n)=\frac{2^{\pi\left(2^{n}\right)}}{2^{1 / n} \prod_{\text {primes } p<2^{n}} p^{1 / n}},
$$

and $\pi$ is the prime counting function. Later it will be convenient to re-write

$$
f(n)=\exp \left[\left(\pi\left(2^{n}\right)-1 / n\right) \log 2-\theta\left(2^{n}\right) / n\right],
$$

where $\theta$ is the Chebyshev function.

Proof. Tatuzawa's theorem [13], says that with at most one exception

$$
\frac{\pi \cdot h(-D)}{\sqrt{D}}=L\left(1, \chi_{-D}\right)>.655 \epsilon D^{-\epsilon},
$$

thus

$$
p(-D)=\frac{2 h(-D)}{2^{g}}>\frac{1.31 \epsilon \cdot D^{1 / 2-\epsilon}}{\pi \cdot 2^{g}} .
$$


Hence it suffices to show $2^{g} \leq f(n) D^{1 / n}$. Suppose first that $D$ is not $\equiv 0(\bmod 8)$.

Let $S=\left\{4\right.$, odd primes $\left.<2^{n}\right\}$, so $\sharp S=\pi\left(2^{n}\right)$. Factor $D$ as $q_{1} \cdots q_{g}$ where $q_{i}$ are (absolute values) of coprime primary discriminants, that is, 4 or odd primes, and satisfy $q_{i}<q_{j}$ for $i<j$. Then, for some $0 \leq m \leq g$, we have $q_{1}, \ldots, q_{m} \in S$ and $q_{m+1}, \ldots, q_{g} \notin S$, and thus $2^{n}<q_{i}$ for $i=m+1, \ldots, g$. This implies

$$
\begin{aligned}
2^{g n} & =\underbrace{2^{n} \cdots 2^{n}}_{m} \cdot \underbrace{2^{n} \cdots 2^{n}}_{g-m} \leq 2^{m n} q_{m+1} q_{m+2} \cdots q_{g} \\
& =\frac{2^{m n}}{q_{1} \cdots q_{m}} D \leq \frac{2^{\# S \cdot n}}{\prod_{q \in S} q} \cdot D
\end{aligned}
$$

as we have included in the denominator the remaining elements of $S$ (each of which is $\leq 2^{n}$ ). The above is

$$
=\frac{2^{\pi\left(2^{n}\right) \cdot n}}{2 \prod_{\text {primes } p<2^{n}} p} \cdot D=f(n)^{n} \cdot D .
$$

This proves the theorem when $D$ is not $\equiv 0 \bmod 8$. In the remaining case, apply the above argument to $D^{\prime}=D / 2$; so

$$
2^{g n} \leq f(n)^{n} D^{\prime}<f(n)^{n} D
$$

Examples. If $0<\epsilon<1 / 2$ and $D>\max (\exp (1 / \epsilon)$, $\exp (11.2))$, then with at most one exception

$$
\begin{array}{ll}
p(-D)>0.10199 \cdot \epsilon \cdot D^{1 / 4-\epsilon} & (n=4) \\
p(-D)>0.0426 \cdot \epsilon \cdot D^{3 / 10-\epsilon} & (n=5) \\
p(-D)>0.01249 \cdot \epsilon \cdot D^{1 / 3-\epsilon} & (n=6) \\
p(-D)>0.00188 \cdot \epsilon \cdot D^{5 / 14-\epsilon} & (n=7)
\end{array}
$$

\section{SECOND VERSION}

Lemma 1. If $g \geq 2$,

$$
\log (D)>g \log (g)
$$

Proof. Again, factor $D$ as $q_{1}, \ldots q_{g}$ where the $q_{i}$ are (absolute values) of primary discriminants, i.e. 4,8 , or odd primes. Let $p_{i}$ denote the $i$ th prime number, so we have

$$
\log (D)=\sum_{i=1}^{g} \log \left(q_{i}\right) \geq \sum_{i=1}^{g} \log \left(p_{i}\right) \stackrel{\text { def. }}{=} \theta\left(p_{g}\right)
$$


By [11, (3.16) and (3.11)], we know that Chebyshev's function satisfies $\theta(x)>x(1-1 / \log (x))$ if $x>41$, and that

$$
p_{g}>g(\log (g)+\log (\log (g))-3 / 2) \text {. }
$$

After substituting $x=p_{g}$ and a little calculation, this gives $\theta\left(p_{g}\right)>$ $g \log (g)$ as long as $p_{g}>41$, i.e. $g>13$. For $g=2, \ldots, 13$, one can easily verify the inequality directly.

Remark. The bound $\log (D)>g \log (g)$ is nearly optimal. That is, for every $g$, there exists a fundamental discriminant (although not necessarily negative) of the form

$$
D_{g} \stackrel{\text { def. }}{=} \pm 3 \cdot 4 \cdot 5 \cdot 7 \ldots p_{g}
$$

and

$$
\log \left|D_{g}\right|=\theta\left(p_{g}\right)+\log (2) .
$$

From the Prime Number Theorem we know $\theta\left(p_{g}\right) \sim p_{g}$, so

$$
\log \left|D_{g}\right| \sim p_{g}+\log (2)
$$

while [11, 3.13] shows $p_{g}<g(\log (g)+\log (\log (g))$ for $g \geq 6$.

Let $W(x)$ denote the Lambert $W$-function, i.e. the inverse function of $f(w)=w \exp (w)$ (see [2], [10, p. 146 and p. 348, ex 209]). For $x \geq 0$ it is positive, increasing, and concave down. The Lambert $W$ function is also sometimes called the product log, and is implemented as ProductLog in Mathematica.

\section{Lemma 2.}

$$
D^{-\log (2) / W(\log (D))}<2^{-g}
$$

Proof. The relation $\log (D)>g \log (g)$ is equivalent to

$$
\log (D)>\exp (\log (g)) \log (g)
$$

Thus applying the increasing function $W$ gives, by definition of $W$

$$
W(\log (D))>\log (g)
$$

and applying the exponential gives

$$
\exp (W(\log (D))>g .
$$

The left hand side above is equal to $\log (D) / W(\log (D))$ by the definition of $W$. Thus

$$
\begin{gathered}
-\log (D) / W(\log (D))<-g \\
D^{-\log (2) / W(\log (D))}=2^{-\log (D) / W(\log (D))}<2^{-g} .
\end{gathered}
$$


Applying Lemma 2 to Tatuzawa's theorem we get that

Theorem 2. If $0<\epsilon<1 / 2$ and $D>\max (\exp (1 / \epsilon), \exp (11.2))$, then with at most one exception

$$
p(-D)>\frac{1.31}{\pi} \epsilon D^{1 / 2-\epsilon-\log (2) / W(\log (D))} .
$$

\section{COMPARISON OF THE TWO THEOREMS}

How do the two theorems compare? Canceling the terms which are the same in both, we seek inequalities relating

$$
\frac{D^{-1 / n}}{f(n)} \quad \text { v. } \quad D^{-\log 2 / W(\log D)}
$$

Theorem 3. For every $n$, there is a range of $D$ where the bound from the first version is better than the bound from the second. However, for any fixed $n$ the bound from the second version is eventually better as $D$ increases.

In other words, we claim that for fixed $n$, as a function of $D$,

$$
D^{\log (2) / W(\log (D))-1 / n} \geq f(n)
$$

on a non-empty compact interval of the $D$ axis. Taking logarithms, it suffices to show that

Lemma 3. Let $n \geq 4$. Then

$$
x\left(\frac{\log 2}{W(x)}-\frac{1}{n}\right) \geq \log f(n)
$$

on some non-empty compact interval of positive real numbers $x$.

Proof. Let $g(n, x)=x(\log 2 / W(x)-1 / n)$. Then

$$
\frac{\partial g}{\partial x}=\frac{\log 2}{W(x)+1}-\frac{1}{n} \quad \text { and } \quad \frac{\partial^{2} g}{\partial x^{2}}=\frac{-\log 2 \cdot W(x)}{x(W(x)+1)^{3}}
$$

This shows $g$ is concave down on the positive real numbers and has a maximum at

$$
x=2^{n}(n \log 2-1) / e .
$$

Because of the concavity, all we need to do is show that $g(n, x)>$ $\log f(n)$ at some $x$. The maximum point is slightly ugly so instead we let $x_{0}=2^{n} n \log 2 / e$.

Using $W(x) \sim \log x-\log \log x$, a short calculation shows

$$
g\left(n, x_{0}\right) \sim \frac{1}{e} \cdot \frac{2^{n}}{n} .
$$


By $[12,5.7)]$, a lower bound on Chebyshev's function is

$$
\theta(t)>t\left(1-\frac{1}{40 \log t}\right), \quad t>678407 .
$$

(Since we will take $t=2^{n}$ this is not much restriction: $n>19$.) By [11, (3.4)], an upper bound on the prime counting function is

$$
\pi(t)<\frac{t}{\log t-3 / 2}, \quad t>e^{3 / 2} .
$$

Hence $-\theta\left(2^{n}\right)<2^{n}(1 /(40 n \log 2)-1)$ and so

$$
\begin{aligned}
\log f(n) & =\left(\pi\left(2^{n}\right)-\frac{1}{n}\right) \log 2-\frac{\theta\left(2^{n}\right)}{n} \\
& <\left(\frac{2^{n}}{n \log 2-3 / 2}-\frac{1}{n}\right) \log 2+\frac{2^{n}}{n}\left(\frac{1}{40 n \log 2}-1\right) \\
& \sim \frac{61}{40 \log 2} \cdot \frac{2^{n}}{n^{2}} .
\end{aligned}
$$

Comparing the two asymptotic bounds for $g$ and $\log f$ respectively we see that

$$
\frac{1}{e} \cdot \frac{2^{n}}{n}>\frac{61}{40 \log 2} \cdot \frac{2^{n}}{n^{2}}
$$

for $n \geq 6$; small $n$ are treated by direct computation ${ }^{1}$

Figure 1 shows a log-log plot of the two lower bounds, omitting the contribution of the constants which are the same in both and the terms involving $\epsilon$. (That is, Theorem 1 gives for each $n$ a lower bound $b(D)$ of the form

$$
\begin{gathered}
b(D)=C(n) \epsilon D^{1 / 2-1 / n-\epsilon}, \quad \text { so } \\
\log (b(D))=(1 / 2-1 / n-\epsilon) \log (D)+\log (C(n))+\log (\epsilon) .
\end{gathered}
$$

Observe that for fixed $n$ and $\epsilon$, this is linear in $\log (D)$, with the slope an increasing function of the parameter $n$. What is plotted is actually $(1 / 2-1 / n) \log (D)+\log (C(n))$ as a function of $\log (D)$, and analogously for Theorem 2.) In red, green, and blue are plotted the lower bounds from Theorem 1 for $n=4,5$, and 6 respectively. In black is plotted the lower bound from Theorem 2 .

Examples. The choice $\epsilon=1 / \log \left(5.6 \cdot 10^{10}\right)$ in Theorem 2 shows that $p(-D)>1$ for $D>5.6 \cdot 10^{10}$ with at most one exception. (For comparison, Weinberger [14, Lemma 4] needed $D>2 \cdot 10^{11}$ to get this lower bound.) And, $\epsilon=1 / \log \left(3.5 \cdot 10^{14}\right)$ in Theorem 2 gives $p(-D)>10$

\footnotetext{
${ }^{1}$ To avoid boring the reader we have omitted the details of checking of when the asymptotics 'kick in'.
} 


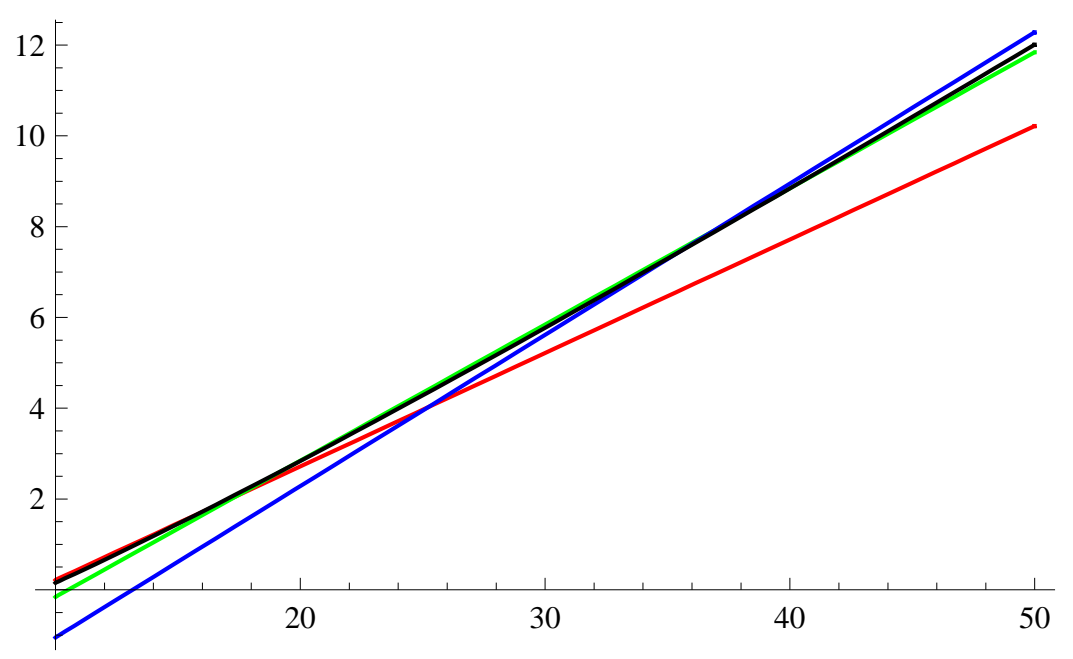

Figure 1. log-log plots of the bounds

for $D>3.5 \cdot 10^{14}$ with at most one exception. On the other hand, $n=6$ and $\epsilon=1 / \log \left(4.8 \cdot 10^{17}\right)$ in Theorem 1 gives $p(-D)>100$ for $D>4.8 \cdot 10^{17}$ with at most one exception.

\section{REFERENCES}

[1] S. Chowla, An extension of Heilbronn's class-number theorem, Quarterly J. Math., 5 (1934), pp. 150-160.

[2] L. Euler, De serie Lambertiana plurimisque eius insignibus proprietatibus, Opera Omnia Ser. 1 Vol. 6, pp. 350-369.

[3] C. F. Gauss, Disquisitiones Arithmeticae, Yale Univ. Press, 1966.

[4] H. Heilbronn, On the class-number in imaginary quadratic fields, Quarterly J. Math., 5 (1934), pp. 304-307.

[5] H. Heilbronn and E. Linfoot, On the imaginary quadratic corpora of classnumber one, Quarterly J. Math., 5 (1934), pp. 293-301.

[6] J. Hoffstein, On the Siegel-Tatuzawa theorem, Acta Arith. XXXVIII (1980), pp. 167-174.

[7] C.G. Ji and H.W. Lu, Lower bound of real primitive $L$-function at $s=1$, Acta Arith. 111 (2004) no. 4, pp. 405-409.

[8] W. Narkiewicz, Elementary and Analytic Theory of Algebraic Numbers, 2nd. ed. Springer-Verlag, 1990.

[9] J. Oesterlé, Nombres de classes des corps quadratiques imaginaires, Sém. Bourbaki, vol. 1983/84, Astérisque, no. 121-122 (1985), pp. 309-323.

[10] G. Pólya and G. Szegö, Aufgaben und Lehrstze der Analysis. Berlin, 1925. Reprinted as Problems and Theorems in Analysis I. Berlin: Springer-Verlag, 1998.

[11] J.B. Rosser and L. Schoenfeld, Approximate formulas for some functions of prime numbers, Illinois J. Math., 6 (1962), pp. 64-94. 
[12] _ Sharper bounds for the Chebyshev functions $\theta(x)$ and $\psi(x)$, Math. Comp., 29 (1975), pp. 243-369.

[13] T. Tatuzawa, On a theorem of Siegel, Jap. J. Math. 21 (1951), pp. 163-178.

[14] P. Weinberger, Exponents of the class groups of complex quadratic fields, Acta Arith. XXII, 1973, pp. 117-124.

Department of Mathematics, University of Texas at Austin, Austin, TX 78712-0257

E-mail address: khopkins@math.utexas.edu

Department of Mathematics, University of California, Santa BarBARA, SANTA BARBARA, CA 93106-3080

E-mail address: stopple@math.ucsb.edu 